\title{
Uplink DPCCH Gating of Inactive UEs in Continuous Packet Connectivity Mode for HSUPA
}

\author{
Tao Chen ${ }^{1}$, Esa Malkamäki ${ }^{2}$, Tapani Ristaniemi ${ }^{3}$ \\ ${ }^{1}$ Nokia Technology Platforms, ${ }^{2}$ Nokia Research Center, ${ }^{3}$ University of Jyväskylä \\ tao.t.chen@nokia.com
}

\begin{abstract}
In order to further improve the packet performance in the UMTS FDD system, uplink DPCCH gating as a scheme is proposed in 3GPP under the work item "Continuous connectivity for packet data users". In this paper, the uplink DPCCH gating concept is described and analyzed on both a qualitative and a quantitative level. And the system performance of different gating patterns used in the scheme was investigated by semianalytical prediction and system level simulations with differen channel profiles. As will be shown, the selection of the gating pattern has a significant influence on the system performance, which is correlated with the uplink data transmission activity and the uplink power control. This contributes to a better understanding of the effects involved with uplink DPCCH gating where the biggest potential for performance optimizations can be found. In summary, the scheme of the uplink DPCCH gating is promising to improve the packet performance.
\end{abstract}

Keywords- DPCCH gating, HSUPA, capacity

\section{INTRODUCTION}

High-speed Uplink Packet Access (HSUPA), which is included in 3GPP Release 6 specification (see, e.g., [1],[2]) has been designed to increase the uplink packet data throughput by means of Hybrid Automatic Repeat reques (HARQ), shorter frame size and fast scheduling controlled by the base station. High Speed Downlink Packet Access (HSDPA) is the downlink counterpart of HSUPA in 3GPP Rel'05 (see, e.g., [3]). Recently, there has been an increasing interest to further improve the packet service in the UMTS FDD system. In 3GPP the improvement of the packet performance is studied under the work item: "Continuous connectivity for packet data users". Several concepts and methods have been proposed under this work item in order to: [4]

- Reduce the uplink noise rise from physical contro channels of packet data users, e.g. for users which have temporarily no data transmission.

- Increase significantly in the UMTS FDD system the number of packet data users (i.e. HS-DSCH/E-DCH users without UL DPDCH) that can stay in CELL DCH state over a long time period, without degrading cell throughput, and that can restart transmission after a period of inactivity with a much shorter delay $(<50 \mathrm{~ms})$ than would be necessary for reestablishment of a new connection.

- Allow improving the achievable UL capacity for VoIP users with its inherent periodic transmission through reducing the overhead of the control channels.
Subsequently, the Continuous Packet Connectivity (CPC) mode as a configuration of the CELL_DCH state has been proposed in 3GPP targeting to Rel'07. The CPC mode, where it is possible to transmit and receive discontinuously (i.e., only in a subset of UL and DL sub-frames), is sufficient especially for the light exchange of data (e.g. VoIP), or during inactive data transfer periods. In addition, as a premise of applying CPC mode, packet data users in CELL_DCH state using HSDPA and HSUPA as described in Rel'06 are addressed.

Uplink DPCCH gating, or discontinuous uplink DPCCH operation, as a candidate scheme applied in CPC mode for HSUPA has been proposed and investigated in 3GPP. In this paper, the gating pattern used in the uplink DPCCH gating as one open issue is studied. Moreover, the packet performance of the uplink DPCCH gating is evaluated on both a qualitative and a quantitative level. And VoIP related performance with uplink DPCCH gating is investigated in [5].

The rest of this paper is organized as follows. In Section II, the principle of uplink DPCCH gating is described, and a realization in a HSUPA scenario is presented. Then, the semianalytical study is performed in Section III. Section IV provides a brief overview of the used simulation model whereas the actual simulation results are presented in section IV. And finally, the conclusions are drawn in Section VI.

\section{UPLINK DPCCH GATING IN A HSUPA SCENARIO}

\section{A. Principle of uplink DPCCH gating}

Previously to 3GPP Rel'06, DPCCH channel was transmitted continuously regardless whether there is actual user data to be transmitted or not, thus highly loading the cell due to the DPCCH overhead of inactive packet users.

Thus, uplink DPCCH gating is proposed targeting to reduce $\mathrm{DPCCH}$ overhead of packet data users. The basic principle in short is that if there is neither E-DCH nor HS-DPCCH transmission, the UE automatically stops the continuous DPCCH transmission and applies a known DPCCH activity (DPCCH on/off) pattern, i.e., the gating pattern, in order to reduce DPCCH overhead of inactive users. With such a solution the inactive packet data users kept in CELL DCH state would not consume any uplink air interface resource. And when an E-DCH or HS-DPCCH transmission takes place also the DPCCH is transmitted regardless of the gating pattern. An example of the uplink DPCCH gating is in Figure 1. 


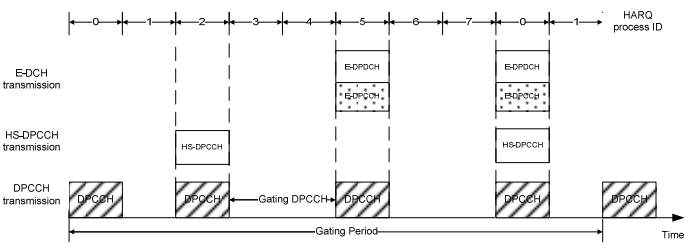

Figure 1. An example of the uplink DPCCH gating

The main advantages of uplink DPCCH gating can be summarized as below:

- Reducing the uplink interference from the physical control channels of inactive packet data users

- Keeping more packet users in Cell_DCH state as long as possible to avoid the signaling delay from the radio link new establishment and state transitions, e.g. the transition between CELL_DCH and CELL_FACH.

- Power saving due to lowered battery consumption, which can result in the longer standby time of the UE.

- Easy operation mode transitions between Rel'06 active mode and CPC mode.

However, due to practical reasons there may be a limit on the length of the DPCCH gating period as during long inactivity the Node B could not any more know whether the uplink synchronization is lost or if there just is a very long inactivity period.

Therefore the actual gating gain depends on several factors: the E-DCH activity, the HS-DPCCH activity and the gating pattern. The E-DCH activity is related with the data traffic and L1 HARQ protocol. And the activity of HS-DPCCH, which carries ACK/NACK messages for the HSDPA L1 HARQ transmissions and Channel Quality Indicator (CQI) messages for the HSDPA scheduling, is dependent on the downlink activity and SRNC configuration. Consequently, the gating pattern as a key parameter of the uplink DPCCH gating scheme shall be set carefully to explore the most gating gain while keeping the uplink synchronization. For example, in the case of high activities on E-DCH/HS-DPCCH, the gating pattern can be set a small value.

\section{B. Realization of uplink DPCCH gating in a HSUPA scenario}

In the realization of uplink DPCCH gating, the key issue is how to perform the uplink power control in the case of the discontinuous uplink transmission, which adjusts the UE transmit power in order to keep the received uplink carrier to interference (CIR) at a given CIR target. During the procedure of the continuous DPCCH transmission, the adjustment to the $\mathrm{DPCCH}$ power is determined by UE based on the previous value that used in the previous slot, whereas in the gating mode, when there is an interruption in transmission, the power of the DPCCH is derived according to the previous value that used in the last slot before the transmission gap. However, after a gating period without tracking the received SIR, the channe response variations could degrade the transmission and increase the interference between the UEs. Consequently, optional power control preambles are defined to be sent previous to the data channel reactivation, which is a period of uplink DPCCH transmission prior to the start of the uplink DPDCH transmission. Besides, the uplink power control during the uplink DPCCH gating can use Power Control Algorithm (PCA) 1 and a 1dB step size as described in [6].

In addition, it is interesting to point out here that the L1 synchronous HARQ protocol specified for HSUPA helps to decrease the impact of inaccuracies in the power control algorithm as illustrated in [7], and thus it will help with the gating technique.

Further, a general scenario of uplink DPCCH gating in HSUPA as shown in Figure 2 is proposed for the study. Both active packet users with the full buffer traffic and inactive users without any data transmission on E-DCH/HS-DPCCH exist simultaneously. The gating pattern is applied on inactive users. Further, the gating pattern modeled here can be also understood as L1 synchronous HARQ transmissions, Preambles, periodical CQI reporting on HS-DPCCH or the transmissions of the periodical service, e.g., VoIP,

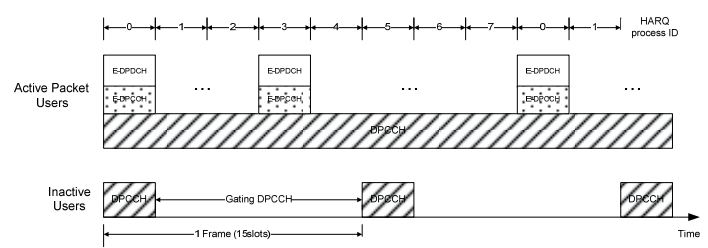

Figure 2. A scenario of uplink DPCCH gating in HSUPA

\section{SEMI-ANALYTICAL STUDY}

In the semi-analytical study, we derive the cell Noise Rise (NR) for the capacity evaluation in HSUPA from the following typical uplink load formula (see, e.g., [8])

$N R_{d B}=-10 \log 10\left(1-\frac{E b N o}{W / R} N \cdot v \cdot(1+i)\right)$,

where $N$ is the number of users per cell, $v$ is the average activity factor, EbNo is the signal energy per bit divided by noise spectral density, $W$ is the chip rate, $R$ is the data rate, $i$ is the other cell to own cell interference ratio, and $N R_{d B}$ is the Noise Rise in dB.

Considering UEs with different activity factors in HSUPA, i.e., different activity factors on physical channels, the NR described above can be further expressed as

$$
N R_{d B}=-10 \log 10\left(1-\sum_{j=1}^{N}\left(E c N o_{c}(j) \cdot v_{c}(j)+E_{c N o}(j) \cdot v_{e}(j)\right) \cdot(1+i)\right),
$$

where $E c N o_{c}(j)$ is the signal energy per chip divided by noise spectral density on DPCCH for the $j$ th UE, $v_{c}(j)$ is the activity factor on DPCCH for the $j$ th $\mathrm{UE}, E b N o_{e}(j)$ is the $E c N o$ on E$\mathrm{DCH}$ for the $j$ th $\mathrm{UE}, v_{e}(j)$ is the activity factor on E-DCH for the $j$ th UE. Consequently, $v_{e}(j)$ would be zero for the inactive UE, which is considered to have the transmission only on $\mathrm{DPCCH}$, i.e., no transmission on E-DCH. However for active 
UEs with the data transmission on E-DCH, $v_{e}(j)$ is a non-zero value. For example, $v_{e}(j)$ would be one for the UE with the full buffer traffic, which may transmit the data every TTI on EDCH associated with DPCCH. In addition, $v_{c}(j)$ shall be one for any UE definitely in Rel'6, whereas can vary with different $\mathrm{DPCCH}$ gating patterns in the CPC mode for the inactive UE. Further, due to the fact that E-DCH resources are always allocated by the scheduling algorithm after the DPCCH resource allocation, the impact of inactive UEs in the CPC mode with only DPCCH transmissions on the capacity can be evaluated based on the NR of inactive UEs, denoted as

$N R_{d B_{-} \text {Inactive }}=-10 \log 10\left(1-N_{\text {inactive }} E c N o_{c} v_{c} \cdot(1+i)\right)$,

where $N_{\text {inactive }}$ stands for the inactive UE number. Note that all inactive UEs have the same $E c N o_{c}$ and $v_{c}$ in order to simplify the study. Therefore, the radio resource allocated to DPCCH transmissions for inactive UEs can be expressed as a function of $N_{\text {inactive }} E c N o_{c}$ and $v_{c}$. Thus, the NR contributed by inactive UEs can be calculated as shown in Figure 3 assuming $i$ is 0.65 and the requirement of $E c N o_{c}$ is about $-18 \mathrm{~dB}$ for the inactive UE.

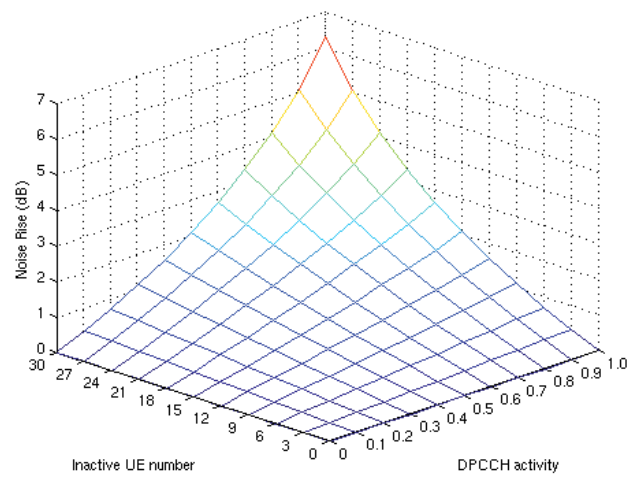

Figure 3. Noise Rise as a function of the inactive UE number and the DPCCH activity factor

Clearly, up to 30 inactive users with the continuous DPCCH transmission can eat up all reserved radio resource, i.e., $6 \mathrm{~dB}$ noise rise target. On the other hand, less DPCCH activity or less inactive UE number can reduce the uplink interference dramatically. In other words, the system can offe more inactive UEs by reducing the DPCCH activity of these UEs. For example, 30 UEs can only lead to $1 \mathrm{~dB}$ noise rise with the DPCCH activity factor of 0.2 , which implies that DPCCH gating in the $\mathrm{CPC}$ mode is a promising approach to reduce the uplink interference, and thus lead to higher capacity and longer staying of the inactive UE in the Cell DCH state. Ideally, fully gating on $\mathrm{DPCCH}$ for inactive UEs can reach the highest capacity gain. Unfortunately, it's impossible to avoid DPCCH transmission totally for the uplink inactive users due to the requirements of the uplink synchronization and TPC commands for the inner loop power control of the downlink FDPCH in Rel'6 or the HS-DSCH associated DCHs in Rel' 5 in the case of the active downlink.

\section{MODELLING AND AssumPTIONS}

\section{A. E-TFC selection}

In WCDMA uplink transmission, UEs have the control of selecting which data rate combination to use. The algorithm for the decision is called TFC elimination/selection for Rel'99 DCH, and E-TFC elimination/selection for the HSUPA EDCH. UEs in CELL_DCH state with an E-DCH transport channel configured shall apply the E-TFC selection procedure when invoked by the HARQ entity. Briefly, E-TFC selection including the scheduling process can be illustrated as below. Firstly, the UE will send Scheduling Requests (SR) in the form of Scheduling Information (SI) and happy bit to Node-B to report the current data buffer, transmission power, etc. SR indicates the UE requesting resource from Node B. Secondly, Node-B will decide the maximum allowed data rate for each UE with the priority function, i.e. scheduling algorithms, based on SIs from UEs, UEs' capabilities and the current interference level. Then Node-B will send Scheduling Grant (SG) signaling to inform the UE the maximum power for the upcoming transmission. Finally, the UE will select the data rate which is suitable for the current status and not requiring higher power than the granted by the Node-B.

\section{B. Channel modeling}

Three types of uplink dedicated physical channels, the uplink DPCCH, the E-DPDCH and the E-DPCCH, are modeled. The DPCCH will carry control information generated at Layer 1, which consists of known pilot bits to support channel estimation for coherent detection, and transmit powercontrol (TPC) commands. Optional feedback information (FBI), and an optional transport-format combination indicator (TFCI) are not assumed here. One E-DPCCH on each radio link is modeled as the overhead. In order to ensure E-DPCCH transmission error free, the power ratio between E-DPCCH and $\mathrm{DPCCH}$, denoted as $\left(\beta_{e c} / \beta_{c}\right)^{2}[6]$, is selected at $3 \mathrm{~dB}$ based on the link level simulation study. One E-DPDCH on each radio link is modeled to carry the E-DCH transport channel. The data transmitted by E-DPDCH will experience the synchronous L1 HARQ, modeled according to [9]. The power ratio between EDPDCH and DPCCH denoted as $\left(\beta_{e d} / \beta_{c}\right)^{2}$ at $8 \mathrm{~dB}$ is applied as an optimized value obtained from the link level simulation study. Therefore, the total transmission power $P_{\text {Total }}$ for the active UEs can be expressed as

$$
P_{\text {Total }}=P_{D P C C H} \cdot\left(1+\left(\frac{\beta_{e c}}{\beta_{c}}\right)^{2}+\left(\frac{\beta_{e d}}{\beta_{c}}\right)^{2}\right),
$$

where $P_{D P C C H}$ is the DPCCH power. Note that the E-DPDCH and E-DPCCH are always frame aligned with each other. However, the total transmission power for inactive UEs will not include E-DCH transmission power.

\section{Environment}

A quasi-static system level simulator, where all necessary RRM algorithms as well as their interactions are modeled, is used to investigate the impact of the DPCCH gating on the capacity. This tool includes a detailed simulation of the users within multiple cells based on the descriptions in [10]. The fast fading is explicitly modeled for each user according to the ITU 
Pedestrian-A profile and ITU Vehicular-A profile. ITU Pedestrian-A channel model is used in these simulations to study the effect of low multipath diversity case. ITU Pedestrian A channel is close to 1-path channel and does not give much multipath diversity while ITU Vehicular A channel gives quite significant multipath diversity. Otherwise, the link leve performance at the system level for the full buffer UE is modeled with Eb/N0 to BLER mapping, called the "Actual Value Interface" (AVI), described in [11].

Main parameters used in the system simulation are summarized in Table I

TABLE I. PARAMETERS SeTtings IN THE SIMULATION

\begin{tabular}{|l|l|}
\hline \multicolumn{1}{|c|}{ Parameter } & \multicolumn{1}{c|}{ Value } \\
\hline Frame Size & $2 \mathrm{~ms}$ TTI (1 Subframe) \\
\hline Inter Site Distance & $2.8 \mathrm{~km}$ \\
\hline Cell Configuration & ITU Veh-A / Ped-A, Macrocell \\
\hline UE Speed & Veh-A 30kmph / Ped-A 3kmph \\
\hline Noise Rise Target & $6 \mathrm{~dB}$ \\
\hline \multicolumn{2}{|c|}{ Active Packet Users Setting } \\
\hline Traffic Model & Full Buffer \\
\hline UE Number & 6 UEs \\
\hline E-TFC Set & $\{64,128,256,384,512,1024\} \mathrm{kbps}$ \\
\hline Number of HARQ Channels & 8 \\
\hline Max Number of L1 Transmissions & 4 \\
\hline$\left(\beta_{e c} / \beta_{c}\right)^{2}$ & $3 \mathrm{~dB}$ \\
\hline Residual FER Target & $1 \%$ \\
\hline \multicolumn{2}{|c|}{ Inactive Users Setting } \\
\hline Traffic Model & No data traffic \\
\hline $\begin{array}{l}\text { DPCCH Gating Pattern } \\
(\text { Gated slots / Total 15slots) }\end{array}$ & NotGated, $9 / 15,12 / 15$ \\
\hline DPCCH CIR Target & $-18.1 \mathrm{~dB}$ \\
\hline Inactive UE Number & $\{0,10,15,20, \ldots, 50\}$ UEs \\
\hline
\end{tabular}

Note that active packet UEs with the full buffer traffic will not have any discontinuous DPCCH transmission. And thereby, the DPCCH gating is only applied on inactive UEs with different gating patterns.

\section{System LeVEl Simulation Results}

The impact of the gating on DPCCH for inactive UEs in the CPC mode, based on the considerations described above, has been evaluated.

Figure 4 and Figure 5 show the significant improvement in the cell throughput with gating DPCCH on inactive UEs for PA3 and VA30 channel profiles separately. Note that ideal reference curves for gating patterns have been calculated simply by scaling the continuous DPCCH curves with the gating ratio. In the case of 20 inactive UEs, the cell throughput from 6 active packet UEs is only $500 \mathrm{kbps}$ without DPCCH gating on inactive UEs, which is just around the half of the cell throughput with the gating pattern of $9 / 15$ on inactive UEs. Moreover, it can be found that more gating on DPCCH will lead to more gain in the cell throughput when comparing cases of the gating pattern $12 / 15$ and $9 / 15$. In addition, it is noted that the performance of the non-gating case is implicitly aligned with the semi-analytical study, i.e., at most about 30 inactive
UEs without the DPCCH gating can be offered in the system assuming $6 \mathrm{~dB}$ NR target.

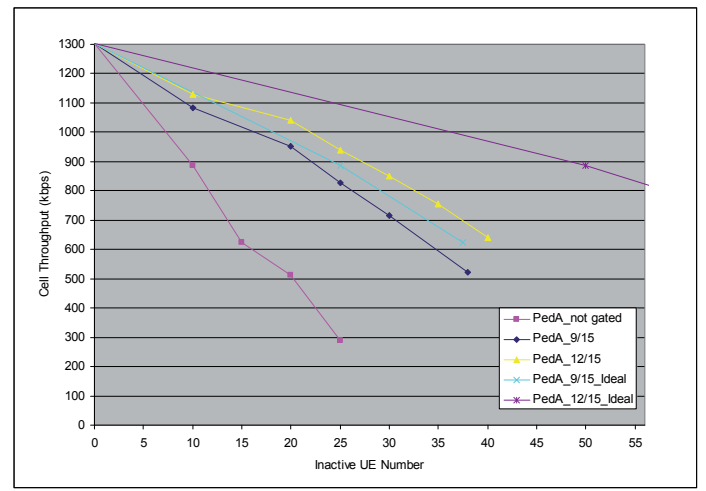

Figure 4. Cell throughput as a function of inactive UEs, different gating patterns vs. ideal gating, PA3

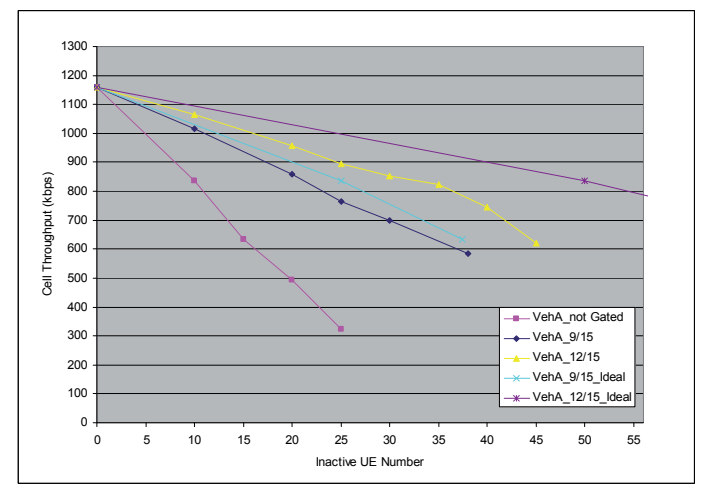

Figure 5. Cell throughput as a function of inactive UEs, different gating patterns vs. ideal gating, VA30

Even if the clear gating gain on the cell throughput can be obtained, unfortunately, it can be limited by the loss from the discontinuous inner loop power control on DPCCH, which may degrade the capability of UE to follow the fast changing channel conditions. Comparing gating cases with and without the ideal power control, this kind of power control loss is shown implicitly. For example, in the case of 40 inactive UEs with the gating pattern $12 / 15$ in PA3, the cell throughput is approximately $800 \mathrm{kbps}$, which is only two third of what it is performed with the ideal power control.

Further, the overshoot, i.e., the probability of noise rise higher than $7 \mathrm{~dB}$ during the simulation, is collected in Figure 6, which shows lower overshoot for gating cases at the same inactive UE number. The overshoot curves for VA30 are flat, whereas PA3 overshoot curves show the fast changing, which implies that ideal power control is more important for PA3 than VA30. In other words, the loss from the DPCCH gating due to the non-ideal power control will less impact VA30 than PA3. 
Thus, more gating on DPCCH for VA30 can be considered in order to reach higher cell throughput.

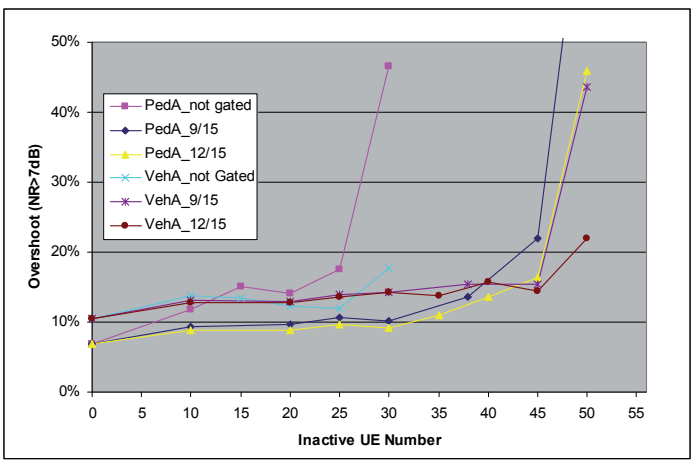

Figure 6. NR Overshoot as a function of inactive UEs, different gating patterns and channel models

On the other hand, more details about the benefit to active UEs from inactive UEs' gating are presented in Figure 7. Clearly, due to the less interference in the uplink, more radio resource can be reserved and allocated to active packet UEs based on the operation of E-TFC selection. Consequently, the high bitrate E-TFC such as $1024 \mathrm{kbps}$ is chosen with the higher probability of up to $35 \%$ for the gating pattern $12 / 15$ than others. Otherwise, the low bitrate E-TFC will be more selected for the non-gating case, e.g, with the probability of more than $60 \%$. Thus, it matches well with the performance of the cell throughput.

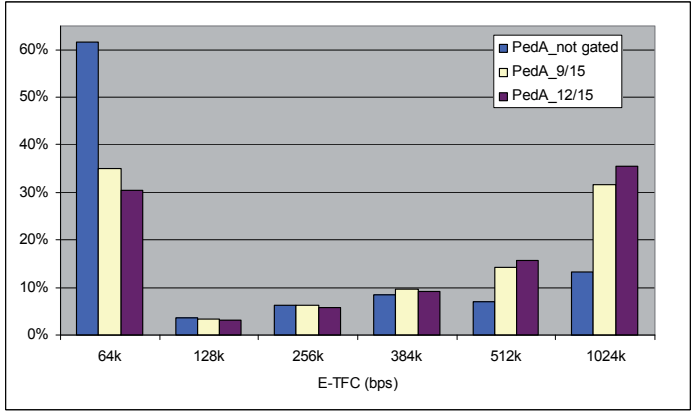

Figure 7. Distribution of E-TFC bitrates of active UEs as a function of the gating pattern with 6 active UEs and 20 inactive UEs per cell, PA3

In order to analyze the performance from the UE point of view, the average UE throughput of the active UE is presented in Figure 8. Approximately $50 \%$ gating gain on the UE throughput can be achieved with the gating pattern $9 / 15$ compared to the non-gating cases, and even more gain for the gating pattern $12 / 15$. Subsequently, the improvement on the UE throughput from the gating is significant as well.

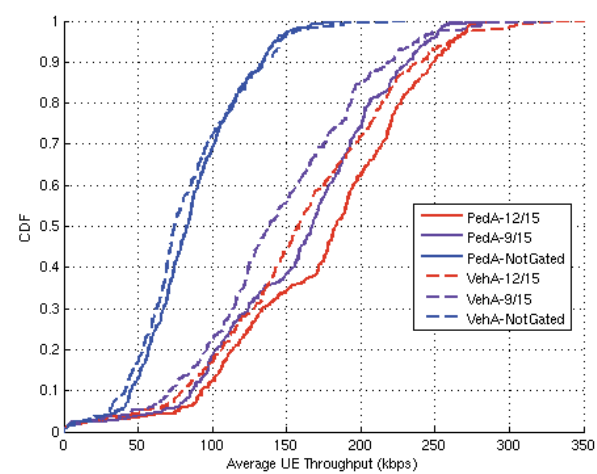

Figure 8. Average UE throughput distribution with 6 active UEs and 20 inactive UEs per cell, different gating patterns and channel models

\section{CONCLUSIONS}

In this paper, we have considered the effects involved with uplink DPCCH gating in CPC mode on the HSUPA system performance. And we analyzed the system performance, which is investigated by semi-analytical prediction and system level simulations. The actual gating gain on the performance depends on the activity in the uplink transmission and the quality of uplink power control. The loss from the uplink power control due to the discontinuous uplink DPCCH transmission may limit the gating gain from the DPCCH gating. And in different channel profiles, the effects of the tradeoff between the DPCCH gating gain and the loss from the discontinuous power control on the system performance can be different, which depends on the requirement on the power control. Therefore, the optimization of the gating pattern should be considered to explore the most gating gain. Generally speaking, the scheme of the uplink DPCCH gating shows a significant improvement on the uplink system performance.

\section{REFERENCES}

[1] 3GPP TS 25.309, "FDD Enhanced Uplink; Overall description; Stage 2"

[2] 3GPP TR 25.896, "Feasibility Study for Enhanced Uplink (FDD)".

[3] 3GPP TS 25.308, "HSDPA; Overall description; Stage2".

[4] 3GPP TR 25.903, "Connectivity for Packet Data Users".

[5] Oscar Fresan, Tao Chen, Esa Malkamäki, Tapani Ristaniemi, "DPCCH Gating Gain for Voice over IP over HSUPA", Submitted to WCNC07.

[6] 3GPP TS 25.214, "Physical layer procedures (FDD)".

[7] Y. Kim, J.-K Han, H. Kwon, D. Kim "Effect of imperfect power control on the performance of HARQ in CDMA2000 reverse link", 15th PIMRC, vol.3,pp.2046-2050,Sept2004.

[8] H. Holma, A. Toskala (Editors), "WCDMA for UMTS", John Wiley and Sons, Third Edition, 2004.

[9] F. Frederiksen, T.E. Kolding, "Performance and Modelling of WCDMA/HSDPA transmission/H-ARQ Schemes", IEEE VTC, Fall, pp.472-481, 2002.

[10] 3GPP2 TSG-C.1002: "1X EV-DV Evaluation Methodology".

[11] Hämäläinen S., P. Slanina, M. Hartman, A. Lappeteläinen, H. Holma, O. Salonaho, "A Novel Interface between Link and System Level Simulations", Proceedings of ACTS summit 1997, Aalborg, Denmark 